
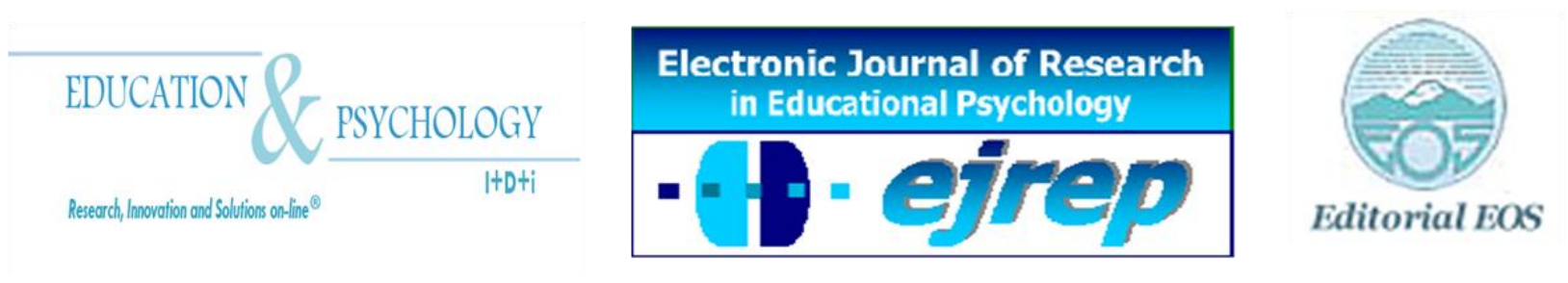

\title{
Perfiles de ansiedad escolar: Diferencias en Clima Social y Violencia entre Iguales
}

\section{María C. Martínez-Monteagudo ${ }^{1}$, Cándido J. Inglés ${ }^{1}$, María V. Trianes ${ }^{2}$ y José M. García-Fernández ${ }^{3}$}

${ }^{1}$ Área de Psicología Evolutiva y de la Educación. Departamento de Psicología de la Salud. Universidad Miguel Hernández de Elche, España.

${ }^{2}$ Departamento de Psicología Evolutiva y de la Educación. Universidad de Málaga, España.

${ }^{3}$ Área de Psicología Evolutiva y de la Educación. Departamento de Psicología Evolutiva y Didáctica. Universidad de Alicante, España.

\section{España}

Correspondencia: Dr. Cándido J. Inglés. Área de Psicología Evolutiva y de la Educación. Departamento de Psicología de la Salud. Universidad Miguel Hernández de Elche. Avda. de la Universidad, s/n. 03202 Elche, Alicante, España. Telefono: +34 9666586 00; ext.: 90 46. Fax: +34 9666589 04. E-mail: cjingles@ umh.es.

(C) Education \& Psychology I+D+i and Editorial EOS (Spain) 


\section{Resumen}

Introducción. La ansiedad escolar es definida como un conjunto de síntomas agrupados en respuestas cognitivas, psicofisiológicas y motoras emitidas por un individuo ante situaciones escolares que son percibidas como amenazantes y/o peligrosas. Entre variables escolares relevantes, la ansiedad escolar puede estar influida por la percepción del clima social y la violencia cotidiana observada en el centro por parte del alumnado. El objetivo de este estudio fue identificar si existen combinaciones entre las distintas situaciones que provocan ansiedad escolar dando lugar a diferentes perfiles ansiosos. Además, se persigue comprobar si existen diferencias entre estos perfiles en las variables percepción de clima social y violencia entre iguales.

Método. La muestra estuvo compuesta por 365 alumnos de educación secundaria. La ansiedad escolar fue medida con el Inventario de Ansiedad Escolar, el clima social se midió con el Cuestionario de Clima Social del Centro Escolar y la violencia entre iguales mediante el Cuestionario de Violencia Escolar Cotidiana.

Resultados. Los análisis de conglomerados identificaron tres perfiles de ansiedad escolar. Los resultados indicaron diferencias estadísticamente significativas entre los tres perfiles en las variables clima referente al profesorado entre los grupos con baja y alta ansiedad escolar y en experiencia personal de sufrir violencia entre el grupo con baja y media ansiedad escolar y entre el grupo con baja y alta ansiedad escolar. La magnitud de las diferencias halladas fue de baja a moderada.

Discusión y Conclusiones. Los hallazgos de este estudio resultan relevantes ya que aportan un análisis más exhaustivo de la ansiedad escolar y de las diferencias observadas en otras variables relevantes en el ámbito educativo, como son el clima social del centro escolar y la violencia entre iguales.

Palabras Clave: ansiedad escolar, clima social, violencia entre iguales, adolescencia. 


\title{
Profiles of School Anxiety: Differences in Social Climate and Peer Violence
}

\begin{abstract}
Introduction. School anxiety is defined as a set of symptoms grouped into cognitive, psychophysiological and motor responses emitted by a subject in school situations that are perceived as threatening and / or dangerous. Between school variables relevant, school anxiety may be influenced by the perception of social climate and the daily violence observed by the students in the school. The aim of this study was to identify whether there are different combinations of school anxiety-provoking situations leading to different anxious profiles. Furthermore, it claims to verify whether there are differences between these profiles on the variables of perception of social climate and peer violence.
\end{abstract}

Method. The sample consists of 365 high school students. School anxiety was measured with the School Anxiety Inventory; the social climate was measured with the School Social Climate Questionnaire and peer violence with the Daily School Violence Questionnaire.

Results. Cluster analyses have identified three profiles of school anxiety. The results indicated statistically significant differences between the three profiles in relation to the climate variables related to teachers between groups with low and high school anxiety and personal experience of suffering violence between groups with low and middle school anxiety among the group with low and high school anxiety. The magnitude of the differences found was low to moderate.

Discussion and Conclusion. The findings of this study are relevant because they provide a more comprehensive analysis of school anxiety and differences in other relevant variables in education, such as the school social climate and peer violence.

Keywords: school anxiety, social climate, peer violence, adolescence.

Received: 06/27/11

Initial acceptance: 06/30/10

Final acceptance: 09/30/11 


\section{Introducción}

La ansiedad escolar es definida como un conjunto de síntomas agrupados en respuestas cognitivas, psicofisiológicas y motoras emitidas por un individuo ante situaciones escolares que son percibidas como amenazantes y/o peligrosas (García-Fernández, Inglés, MartínezMonteagudo y Redondo, 2008). Los estudios epidemiológicos indican que es relativamente frecuente y pueden afectar hasta el 18\% de los niños y adolescentes entre 3 y 14 años (Bados, 2005; Granell de Aldaz, Vivas, Gelfand y Feldman, 1984).

Existe una cantidad ingente de investigaciones que han tratado de identificar, catalogar y definir los miedos de niños y adolescentes en términos generales (Burham, 2005, 2009; Ollendick, 1983; Sandín, 1997; Scherer y Nakamura, 1968; Valiente, Sandín, Chorot y Tabar, 2003) y también, aunque en menor medida, las situaciones generadoras de ansiedad escolar (Méndez, García-Fernández y Olivares, 1996; Morris, Finkelstein y Fisher, 1976; Steinhausen, Müller y Winkler, 2008; Swartz, Reyns, Henson y Wilcox, 2011). Sin embargo, aún existen algunas lagunas en la investigación como, por ejemplo, analizar la relación de la ansiedad escolar con otras variables relevantes del contex to escolar así como también estudiar las diferencias que se producen en estas variables entre los grupos de baja y alta ansiedad escolar.

En general, son numerosas las situaciones que pueden provocar ansiedad en el ámbito escolar (p. ej., jugar durante el recreo, hablar en clase, otros niños, los exámenes, etc.). Así, realizando una amplia revisión de la literatura previa, la ansiedad escolar se podrían agrupar en cuatro grandes categorías: (a) ansiedad ante situaciones de fracaso y castigo escolar; (b) ansiedad ante la agresión; (c) ansiedad ante la evaluación social en el ámbito escolar; y (d) ansiedad ante la evaluación escolar. Del mismo modo, la mayoría de investigaciones que han pretendido evaluar la ansiedad escolar a través de cuestionarios, inventarios o escalas recogen situaciones o ítems que podrían ser clasificados en estos cuatro apartados. Queda patente que, en términos generales, éstas son las situaciones escolares más frecuentes según la literatura científica. Además, en general, los estudios han hallado correlaciones significativas entre las situaciones generadoras de ansiedad escolar, concluyendo la existencia de una estrecha relación entre ellas (Dunn, 1970; García-Fernández, Espada, Orgilés y Méndez, 2010; GarcíaFernández, Inglés, Martínez-Monteagudo, Marzo y Estévez, 2011; Méndez, 1988). 
Entre variables escolares relevantes, la ansiedad escolar puede estar influida por la percepción del clima social y la violencia cotidiana observada en el centro por parte del alumnado (Astor, Benbenishty, Zeira y Vinokur, 2002; Martínez-Rodríguez, 2004; Thomas, 2010). A su vez, estas dos variables han sido estudiadas ampliamente en la literatura científica (Castro y Gaviria, 2005; Espinoza, 2006; Fernández-Baena et al., 2011; Gázquez, Pérez y Carrión, 2011; Guil y Mestre, 2004; Infante et al., 2003; Moreno, Estévez, Murgui y Musitu, 2009; Sánchez, Rivas y Trianes, 2006). En lo que respecta al clima escolar, los autores se han centrado principalmente en dos ámbitos de estudio: clima académico y clima social de la clase. El primero se refiere al grado en que el entorno de aprendizaje estimula el esfuerzo y enfatiza la cooperación (Roeser y Eccles, 1998) y el segundo se define como la calidad de las interacciones entre estudiantes-profesores y entre estudiantes-estudiantes (Emmons, Comer y Haynes, 1996), o como la percepción por parte de alumnos y profesores de bienestar personal, sentimientos positivos de sentirse aceptado y ser valioso para los demás en la convivencia diaria (Trianes, 2000). Un clima social adecuado presenta efectos positivos sobre el ajuste psicológico (Kuperminc, Leadbeater y Blatt, 2001; Roeser y Eccles, 1998), sobre todo en estudiantes que pueden tener riesgos de dificultades académicas, emocionales o comportamentales (Felner et al., 1995; Haynes, Emmons y Ben-Avie, 1997), asociándose a un desarrollo saludable, un aprendizaje óptimo y disminuyendo las conductas desadaptativas (Kuperminc, Blatt y Leadbeater, 1997; Ladd, 1990; Parker y Asher, 1987; Westling, 2002). Otras intervenciones que mejoran el clima social encuentran también beneficios en la adaptación escolar y social (Comer, Ben-Avie, Haynes y Joyner, 1999; Romasz, Cantor y Elias, 2004) y en la capacidad de afrontamiento, autoconcepto y autoestima, empatía y sociabilidad (Aciego, Domínguez y Hernández, 2003). Atendiendo a estas investigaciones, el clima social escolar podría influir en los niveles de ansiedad escolar. Sin embargo, en la actualidad no se cuenta con estudios relevantes que aclaren esta relación.

Por otro lado, actualmente se tiene en cuenta la violencia interpersonal entre iguales de bajo o moderado impacto, la cual ocurre cuando una persona o grupo de personas se ve insultada, físicamente agredida, socialmente excluida o aislada, amenazada o atemorizada por otro/s de forma puntual y no reiterada (Ortega, del Rey y Mora-Merchán, 2001). Este fenómeno ha sido denominado violencia cotidiana entre iguales, refiriéndose a aquellos entornos caracterizados por relaciones interpersonales que resuelven los conflictos mediante actos agresivos injustificados, recíprocos en su mayoría, de intensidad leve o media y alta frecuencia entre el alumnado (Fernández-Baena et al., 2011). Investigaciones recientes muestran que 
sufrir este tipo de violencia, sin episodios de acoso o maltrato, también puede predecir inadaptación personal y psicopatología (Cangas, Gázquez, Pérez-Fuentes, Padilla y Miras, 2007; Trianes, 2004).

\section{Objetivos e hipótesis}

Teniendo en cuenta estos planteamientos, con este trabajo se pretende lograr básicamente dos objetivos. En primer lugar, se persigue comprobar si existen diferentes perfiles de alumnos con ansiedad escolar. En segundo lugar, una vez hallados y definidos los perfiles, se intenta averiguar si existen diferencias significativas entre los grupos identificados respecto de algunas variables del ámbito educativo seleccionadas, como son el clima social y la violencia cotidiana del centro.

A partir de la evidencia empírica previa, se espera que: (a) las combinaciones de las situaciones de ansiedad escolar den lugar a diferentes perfiles ansiosos y (b) existan diferencias estadísticamente significativas entre los perfiles de ansiedad escolar con respecto al clima referente al centro, clima referente al profesorado, experiencia personal de sufrir violencia y violencia observada en el centro.

\section{Método}

\section{Participantes}

El total de participantes en la investigación fue de 452 estudiantes de $1^{\circ}$ de ESO a $4^{\circ}$ de ESO de la ciudad de Málaga (España), de los que 52 (16.35\%) fueron excluidos por omisiones o errores en sus respuestas o por no obtener el consentimiento informado por escrito de sus padres para participar en la investigación y 35 (5.02\%) fueron excluidos por ser extranjeros con importantes déficits en el dominio de la lengua española. Por tanto, la muestra se compuso finalmente de 365 estudiantes, con un rango de edad de 12 a 16 años $(M=13.71$; $D T=1.47)$. La distribución de la muestra fue la siguiente: $1^{\circ}$ ESO (57 varones y 53 mujeres), $2^{\circ}$ ESO (65 varones y 46 mujeres), $3^{\circ}$ ESO (36 varones y 25 mujeres) y $4^{\circ}$ ESO (52 varones y 31 mujeres). La prueba Chi-cuadrado de homogeneidad de la distribución de frecuencias reveló la ausencia de diferencias estadísticamente significativas entre los ocho grupos Sexo x Curso $\left(\chi^{2}=2.46 ; p=.482\right)$. 


\section{Instrumentos}

Inventario de Ansiedad Escolar, IAES. El IAES (García-Fernández et al., 2011) es un instrumento que evalúa las situaciones y respuestas de ansiedad en alumnos de ESO y Bachillerato (12 a 18 años), mediante tres escalas de respuestas de ansiedad y cuatro factores situacionales. El alumno tiene que indicar en un rango de 0 a $4(0=$ nunca; $4=$ siempre $)$ la frecuencia con que se da cada respuesta en la situación a la que hace referencia. A mayor puntuación, mayor ansiedad escolar.

Los cuatro factores situacionales están formados por 23 situaciones escolares a las cuales el alumno debe contestar en función de sus repuestas cognitivas, conductuales y psicofisiológicas. Estos factores son: (a) Ansiedad ante el Fracaso y Castigo Escolar (AFCE): incluye 8 ítems relativos a situaciones escolares de fracaso escolar (e.g., "suspender un examen") y de castigo (e.g., "ser enviado al director"), (b) Ansiedad ante la Agresión (AA): consta de 6 ítems referidos a la ansiedad ante la posibilidad de sufrir agresiones físicas o verbales por parte de los iguales (e.g., "que me insulten o amenacen"), (c) Ansiedad ante la Evaluación Social (AES): compuesto por 5 ítems que evalúan miedos de naturaleza social relacionados con la actuación en público (e.g., "hablar a la clase”) y (d) Ansiedad ante la Evaluación Escolar (AEE): formado por 4 ítems relativos a situaciones en donde se evalúan las competencias escolares (e.g., "hacer un examen escrito").

Las factores relativos a los tres sistemas de respuesta son: (a) Ansiedad Cognitiva (AC): compuesto por 9 ítems que indican pensamientos, sentimientos, etc., ante las distintas situaciones escolares (e.g., "siento temor a equivocarme"), (b) Ansiedad Conductual (ACO): incluye 5 ítems que reflejan aspectos conductuales fácilmente observables ante dichas situaciones (e.g., "me tiembla la voz") y (c) Ansiedad Psicofisiológica (AP): formado por 5 ítems que evalúan la activación del sistema nervioso ante las mismas situaciones (e.g., "siento molestias en el estómago").

Los análisis factoriales exploratorios y confirmatorios realizados por GarcíaFernández et al. (2011) apoyaron la estructura de cuatro factores situacionales correlacionados los cuales explicaron el 74,97\% de varianza total, así como la estructura de tres factores relativos a los sistemas de respuesta de la ansiedad que explicaron el 68,64\%, el 67,70\% y el 
$58,51 \%$ de la varianza total relativos a la ansiedad cognitiva, psicofisiológica y conductual, respectivamente.

Los coeficientes de consistencia interna (alfa de Cronbach) para las puntuaciones del IAES fueron: .93 (AES), .92 (AFCE y AA), .88 (AEE), .86 (AC y AP) y .82 (ACO). La fiabilidad test-retest, para un intervalo de 2 semanas, fue: .84 (AFCE y AES), 83 (AEE), .78 (AA), .77 (AC), .75 (AP) y .74 (ACO). En este estudio únicamente se utilizaron las puntuaciones obtenidas en los cuatro factores situacionales del IAES. La validez concurrente fue examinanda mediante coeficientes de correlación entre el IAES y el Inventario de Ansiedad Estado-Rasgo (STAI; Spielberg, Gorsuch y Lushene, 1970), hallándose correlaciones positivas y estadísticamente significativas.

Cuestionario de Clima Social del Centro Escolar, CECSCE (Trianes, Blanca, de la Morena, Infante y Raya, 2006). El CECSCE evalúa el clima escolar relativo al centro y relativo al profesorado mediante 14 ítems. El relativo al centro se refiere al grado en que el entorno de aprendizaje estimula el esfuerzo y enfatiza la cooperación. El relativo al profesorado evalúa la calidad de las interacciones entre estudiantes-profesores, la percepción por parte de alumnos y profesores de bienestar personal y los sentimientos positivos de sentirse aceptado y ser valioso para los demás en la convivencia diaria. Se trata, pues, de un cuestionario que se centra en las relaciones interpersonales entre estudiantes y profesores. Consta de dos factores: (a) Clima referente al Centro (CC): consta de 8 ítems que reflejan el clima social del centro escolar, evaluando la percepción de los alumnos de comportamientos de ayuda, respeto, seguridad y confort en el centro. Un ítem ejemplo sería "cuando hay una emergencia, hay alguien para ayudarme" o "mi instituto es un lugar muy seguro"; y (b) Clima referente al Profesorado (CP): Contiene 6 ítems referentes a la relación alumno-profesor. Representa percepciones de satisfacción en la relación con el profesorado, analizando su actuación según valores de justicia y respeto (e.g., "los profesores de este centro son agradables con los estudiantes" o "cuando los estudiantes rompen las reglas son tratados justamente").

Los resultados muestran un alfa de .77 para el factor CC, y .72 para el factor CP. El intervalo entre test y el retest fue de 9 meses, obteniendo una correlación entre ambas mediciones de .61 . 
Cuestionario de Violencia Escolar Cotidiana, CUVECO (Fernández-Baena et al., 2011). EL CUVECO está construido a partir de los ítems del California School Climate and Safety Survey (CSCSS; Rosenblatt y Furlong, 1997). La escala evalúa aspectos demográficos, percepción del clima escolar, seguridad, apoyo social, deseabilidad social, victimización de violencia escolar y hostilidad. Los ítems se presentan en formato de respuesta tipo Likert de cinco puntos $(1=$ nunca y $5=$ casi siempre $)$.

Para analizar la estructura factorial del CUVECO se llevaron a cabo varios análisis factoriales de componentes principales con rotación oblimin. Con objeto de estudiar la estabilidad de la solución factorial encontrada, la muestra fue dividida de forma aleatoria en dos mitades, realizándose el análisis factorial en cada uno de los grupos resultantes. Finalmente, se efectuó el análisis factorial con la muestra total, coincidiendo la solución factorial hallada con las anteriores, e incluyendo los mismos ítems y saturaciones aproximadas.

Se obtuvieron dos factores que explican que explican el $33.31 \%$ y el $15.51 \%$ de la varianza respectivamente. El primer factor denominado Experiencia Personal de Sufrir Violencia (EPSV) está compuesto por 8 ítems que reflejan las agresiones de tipo físico, verbal o psicológico que padece el alumnado víctima de la violencia cotidiana entre iguales, con saturaciones positivas y superiores a .30 en todos los elementos (e.g., "me han dado puñetazos o patadas"). El segundo factor denominado Violencia Observada en el Centro (VOC) contiene 6 ítems referentes principalmente a comportamientos violentos entre iguales que son observados por los estudiantes en el centro educativo, con saturaciones también altas y positivas en todos los ítems (e.g., "los estudiantes se meten en peleas"). Ambos factores presentan una correlación significativamente baja con un valor de .31.

Los coeficientes de consistencia interna (alfa de Cronbach) para las puntuaciones del CUVECO fueron: .85 (EPSV) y .74 (VOC). El intervalo entre test y retest fue de seis meses dentro de un mismo curso escolar, obteniéndose una correlación entre ambas mediciones de 63.

Para el estudio de la validez convergente se relacionaron las puntuaciones obtenidas en el CUVECO con las obtenidas en el Cuestionario de Clima Social del Centro Escolar, CECSCE (Trianes et al., 2006). Así, las puntuaciones en el factor de experiencia personal se asociaron de forma significativa $(p<.01)$ con la medida de clima social referente al centro $(r=$ 
-.15), pero no así con el clima referente al profesorado. Por su parte, en las puntuaciones de violencia observada se apreciaron asociaciones significativas $(p<.01)$ con la medida de clima social referente al centro $(r=-.34)$ y con el clima referente al profesorado $(r=-.18)$.

\section{Procedimiento}

Se llevó a cabo una entrevista con los directores, jefes de estudios y/o jefes del Departamento de Orientación de los centros participantes, para exponer los objetivos de la investigación, describir los instrumentos de evaluación, solicitar permiso para su aplicación y promover su colaboración. Los cuestionarios fueron administrados colectivamente en el aula por los investigadores. A continuación se leyeron en voz alta las instrucciones, resaltando la importancia de no dejar ninguna pregunta sin contestar. Los investigadores estuvieron presentes durante la administración de las pruebas para aclarar posibles dudas y verificar la cumplimentación independiente por parte de los alumnos. El tiempo medio de aplicación de las pruebas fue de 25 minutos para el IAES y de cinco minutos para el CECSCE y el CUVECO, respectivamente.

\section{Análisis de datos}

Con el objetivo de identificar los perfiles de ansiedad escolar de los estudiantes se recurrió al análisis de conglomerados (método quick cluster analysis). Dentro del análisis de conglomerados existen dos grandes categorías: métodos jerárquicos y no jerárquicos. Los más utilizados para este tipo de estudios, son los primeros. La principal diferencia entre los métodos jerárquicos y no jerárquicos es que en los últimos el investigador debe especificar a priori los grupos que deben ser formados. En este caso se ha utilizado el "quick cluster analysis", el cual además de ser un método no jerárquico también es un método de reasignación, es decir, permite que un individuo asignado a un grupo en un determinado paso del proceso, sea reasignado a otro grupo en un paso posterior si esto optimiza el criterio de selección. Este análisis de conglomerados es el procedimiento más adecuado para establecer perfiles en una muestra amplia de sujetos (Hair, Anderson, Tatham y Black, 1998).

Los perfiles de ansiedad escolar se han definido a partir de las diferentes combinaciones de los cuatro factores situacionales (Ansiedad ante el Fracaso y Castigo Escolar, Ansiedad ante la Agresión, Ansiedad ante la Evaluación Social y Ansiedad ante la Evaluación Escolar) que evalúa el IAES (García-Fernández et al., 2011). Para eliminar el efecto debido a las diferencias en la medida de la ansiedad escolar (debido a que el número de ítems de cada una de 
las subescalas no es el mismo), se ha realizado el análisis de conglomerados después de haber estandarizado las puntuaciones directas.

El criterio seguido en la elección del número de conglomerados fue el maximizar las diferencias inter-clusters con el fin de conseguir establecer el mayor número posible de grupos con diferentes combinaciones. Una vez identificados los perfiles de ansiedad escolar, mediante análisis de varianza (ANOVA) se analizó la significación estadística de las diferencias existentes entre los grupos en la percepción del clima referente al centro, del clima referente al profesorado, experiencia personal de sufrir violencia y violencia observada en el centro. Puesto que el factor está integrado por más de dos niveles o grupos se utilizó como prueba de comparaciones múltiples post-hoc la prueba de Scheffé. Todos los análisis estadísticos se han realizado con el programa SPSS 16.0.

\section{Resultados}

Identificación de perfiles de ansiedad escolar

Los resultados del análisis de conglomerados permitieron diferenciar tres grupos caracterizados por distintos niveles de intensidad en los cuatro factores situacionales de ansiedad escolar (véase Figura 1).

Un primer grupo, compuesto por 141 estudiantes (38.63\%), se caracteriza por puntuaciones bajas en todos los factores evaluados. Este grupo se ha definido por un perfil con baja ansiedad escolar (Grupo BAE). El segundo grupo, integrado por 163 alumnos (44.66\%), se caracteriza por puntuaciones medias en la totalidad de los factores. Este grupo se ha definido con media ansiedad escolar (Grupo MAE). Por último, el tercer grupo formado por 61 estudiantes $(16.71 \%)$, está caracterizado por puntuaciones altas en los cuatro factores situacionales. Este grupo se ha definido por un perfil con alta ansiedad escolar (AAE). 


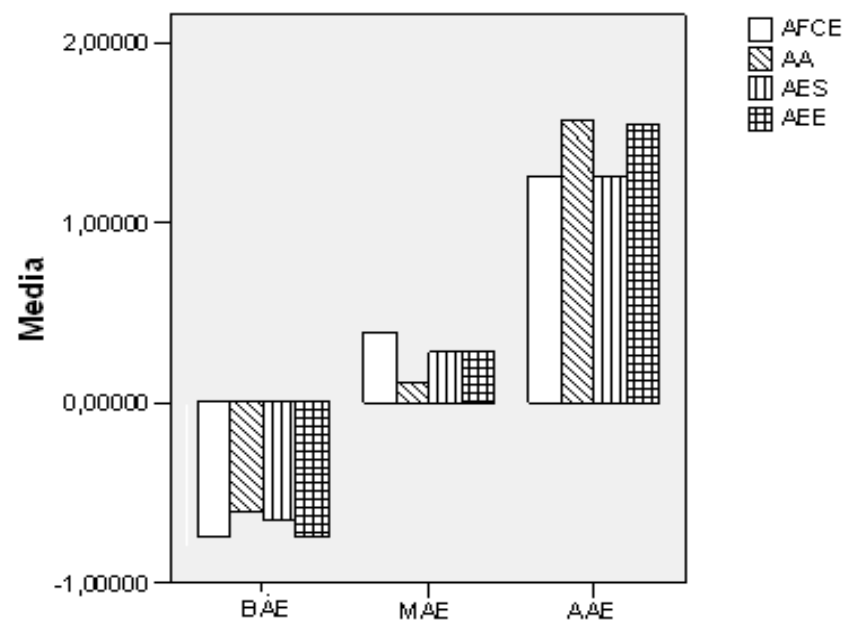

Figura 1. Representación gráfica de los perfiles de ansiedad escolar identificados a través del análisis de conglomerados

AFCE: Ansiedad ante el Fracaso y Castigo Escolar; AA: Ansiedad ante la Agresión; AES: Ansiedad ante la Evaluación Social; $A E E$ : Ansiedad ante la Evaluación Escolar; $B A E$ : Baja Ansiedad Escolar; MAE: Moderada Ansiedad escolar; AAE: Alta Ansiedad Escolar.

\section{Diferencias intergrupo}

Los resultados del ANOVA indican la existencia de diferencias estadísticamente significativas entre los tres perfiles de ansiedad escolar en las variables clima referente al profesorado y experiencia personal de sufrir violencia (véase Tabla 1).

Las comparaciones post-hoc (Prueba de Scheffé) revelan que dichas diferencias se producen entre el Grupo BAE y el Grupo AES en la variable clima referente al profesorado, obteniendo el perfil con alta ansiedad escolar mayores puntuaciones en esta variable. La magnitud de la diferencia hallada es moderada $(p=.003, \mathrm{~d}=-.51)$. De este modo, los alumnos con una alta ansiedad escolar tienen mejor percepción del clima respecto a su profesorado que los alumnos con una baja ansiedad escolar.

Con respecto a la variable experiencia personal de sufrir violencia, los resultados indican que existen diferencias estadísticamente significativas entre el Grupo BAE y el Grupo MAE $(p=.011, \mathrm{~d}=-.39)$ y entre el Grupo BAE y el Grupo AES $(p=.010, \mathrm{~d}=-.39)$. No obs- 
tante, la magnitud de las diferencias es baja en ambos casos. Así, los sujetos incluidos en los grupos caracterizados por una moderada y alta ansiedad escolar han sufrido episodios de violencia escolar en mayor medida que los alumnos del grupo con baja ansiedad escolar (Véase Tabla 1).

Tabla 1. Diferencias en perfiles de ansiedad escolar en variables analizadas

\begin{tabular}{lccccc}
\hline & BAE & MAE & AES & \multicolumn{2}{c}{$\begin{array}{c}\text { Significación } \\
\text { estadística }\end{array}$} \\
\hline Muestra total & $M(D T)$ & $M(D T)$ & $M(D T)$ & $F_{(2.362)}$ & $p$ \\
\hline CC & $25.99(4.99)$ & $26.30(4.94)$ & $25.67(5.59)$ & .371 & .690 \\
CP & $20.81(4.14)$ & $21.60(3.67)$ & $22.86(3.76)$ & 6.051 & .003 \\
EPSV & $11.41(3.90)$ & $13.09(5.15)$ & $13.16(5.68)$ & 5.403 & .005 \\
VOC & $16.85(5.21)$ & $17.22(5.43)$ & $18.13(5.31)$ & 1.215 & .298 \\
\hline
\end{tabular}

$C C$ : Clima referente al Centro; $C P$ : Clima referente al Profesorado; EPSV: Experiencia Personal de Sufrir Violencia; VOC: Violencia Observada en el Centro; BAE: Baja Ansiedad Escolar; $M A E$ : Moderada Ansiedad Escolar; $A A E$ : Alta Ansiedad Escolar.

\section{Discusión y Conclusiones}

El objetivo de este estudio fue identificar grupos de distinta intensidad de ansiedad escolar autoinformada por los estudiantes. Posteriormente, se pretendía comprobar si existen diferencias estadísticamente significativas entre estos grupos en las variables clima referente al centro, clima referente al profesorado, experiencia personal de sufrir violencia y violencia observada en el centro.

Mediante el análisis de conglomerados se han identificado tres perfiles de ansiedad escolar. Un primer grupo con un perfil de baja ansiedad escolar (Grupo BAE), un segundo grupo con un perfil de media ansiedad escolar (Grupo MAE) y, por último, un tercer grupo con un perfil de alta ansiedad escolar (Grupo AES). En este resultado ha predominado la intensidad de la respuesta de ansiedad que ha producido tres niveles. Así, frente a otras combinaciones más cualitativas, ha predominado el aspecto intensivo, revelando que en la ansiedad, 
quizás porque su naturaleza emocional genera relaciones curvilineales con otras variables, se discrimina el grupo con niveles saludables (bajos) del que tiene niveles amenazantes (altos) para la salud psicológica. El grupo medio adopta un papel diferente según la variable estudiada. Otra experiencia cercana como es la del estrés, también produce resultados diferenciales para el grupo bajo saludable.

Los tres grupos obtenidos según la graduación de intensidad de la ansiedad presentan diversas relaciones con las otras variables del estudio, permitiéndonos mantener así la primera de nuestras hipótesis. Estos grupos presentan diferencias en las variables percepción del profesorado y en episodios de sufrir violencia en el centro. Así, los estudiantes que presentan un perfil de alta ansiedad escolar presentan diferencias significativas con respecto al grupo de baja ansiedad escolar en lo que se refiere a la percepción del profesorado. De modo que los alumnos con alta ansiedad escolar tienen una mejor percepción de su profesorado con respecto a su actuación en términos de calidez y apoyo. En la actualidad, no existen investigaciones previas que permitan comparar dichos resultados. Una razón que puede explicar, en parte, estos resultados se refiere a que, debido a su problema de ansiedad, el alumnado más ansioso haya podido recibir más ayuda de su profesorado, ya que la ansiedad es percibida como problema que suele ser detectado y atendido desde la institución escolar (Monjas, 2001). Otra explicación posible de los resultados hallados podrían estar indicándonos como los alumnos con alta ansiedad escolar presentan un mayor respeto por las figuras de autoridad, y concretamente por el profesorado, ya que éstos les proporcionan un sentimiento de seguridad y protección frente a las exigencias y dificultades del curso académico en la educación secundaria.

Por otro lado, los alumnos con un perfil moderado y alto de ansiedad escolar han sufrido más episodios personales de violencia que los alumnos con baja ansiedad escolar. Se revela aquí una casuística que afecta al alumnado con problemas de ansiedad, el haber estado implicado en agresiones como víctima y posiblemente también como agresor. Otras investigaciones, trabajando con el estrés, una variable cercana a la ansiedad, encuentran que alumnado con baja aceptación de los iguales (que se produce por conflictos interpersonales y muchas veces por agresividad injustificada) presenta altos niveles de estrés en educación primaria (Escobar, Trianes, Fernández y Miranda, 2010) así como en educación secundaria. También se ha hallado que el alumnado implicado en peleas y conductas agresivas presenta niveles más altos de estrés adolescente (Escobar, Blanca, Fernández-Baena y Trianes, 2011). Estos resultados apuntan a que, una causa o consecuencia asociada a sufrir episodios de violen- 
cia interpersonal en el centro y rechazo asociado es experimentar ansiedad y estrés. Se revela así un riesgo asociado a sufrir violencia y estar implicado en conductas agresivas, consistente en sufrir síntomas de psicopatología internalizada. Este resultado es relativamente novedoso ya que la mayor parte de los estudios previos encuentran una asociación directa entre estrés y problemas externalizados en población infantil y adolescente (Elgar, Arlett y Groves, 2003; Suldo, Shaunessy, Thalji, Michalowski y Shaffer, 2009; Trianes et al., 2009). No obstante también hay autores que han encontrado asociación entre violencia escolar sufrida y ansiedad en sus diversas manifestaciones (Ezpeleta, 2005; Hawker y Boulton, 2000).

Por otro lado, no han sido halladas diferencias estadísticamente significativas en el resto de variables analizadas. Así, aunque diversas investigaciones han relacionado el término ansiedad escolar con el clima social del aula o con la violencia interpersonal (Astor et al., 2002; Martínez-Rodríguez, 2004; Thomas, 2010), hasta ahora no se había profundizado en las variables que engloban estos constructos con respecto a la ansiedad escolar. De modo, que atendiendo a los resultados, el clima referente al centro y la violencia observada no producen diferencias estadísticamente significativas en los distintos perfiles de ansiedad escolar, siendo, por tanto, las variables más próximas y personales al alumno, como es la relación directa con el profesor o sufrir episodios violentos las que influyen en mayor medida.

El presente estudio presenta diversas limitaciones, la cuales deberían ser remediadas en futuras investigaciones. En primer lugar, la muestra se compuso únicamente de estudiantes de ESO, por lo que los resultados obtenidos en el mismo no pueden generalizarse a estudiantes de otros niveles educativos como Educación Primaria, Bachillerato y Educación Superior. Futuros trabajos deberían confirmar, usando muestras representativas, si los resultados hallados en este estudio difieren o se mantienen en otros niveles educativos con el fin de incrementar la validez externa de estos hallazgos. Además, sería muy recomendable realizar estudios que administren una entrevista clínica u otra metodología de evaluación, para que el diagnóstico no se base exclusivamente en la puntuación de una medida de autoinforme.

A pesar de estas limitaciones, los hallazgos de este estudio resultan relevantes ya que aportan un análisis más exhaustivo de la ansiedad escolar y de las diferencias observadas en otras variables relevantes en el ámbito educativo. Los resultados pueden ser utilizados por profesores, psicólogos escolares y psicólogos clínicos como base empírica para desarrollar acciones preventivas y de intervención más eficaces. Para ello, los profesionales deberían 
disponer de información relativa al perfil de ansiedad escolar que presentan sus alumnos, con el fin de ajustar las intervenciones. Además, los paquetes de intervención dirigidos a la prevención o disminución de la ansiedad escolar deberían considerar el papel de las variables clima social y violencia entre iguales. En cualquier caso, esta cuestión debería ser objeto de un análisis más profundo en futuras investigaciones.

\section{Referencias}

Aciego, R., Domínguez, R. y Hernández, P. (2003). Evaluación de la mejora en valores de realización personal y social en adolescentes que han participado en un programa de intervención. Psicothema, 15(4), 589-594.

Astor, R. A., Benbenishty, R., Zeira, A. y Vinokur, A. (2002). School climate, observed risky behaviors, and victimization as predictors of high school students' fear and judgments of school violence as a problem. Health Education and Behavior, 29(6), 716-736.

Bados, A. (2005). Trastorno de ansiedad por separación. Rechazo escolar y fobia escolar. Facultad de Psicología. Universidad de Barcelona.

Burnham, J. J. (2005). Fears of children in the United States: A detailed examination of the American Fear Survey Schedule. Measurement and Evaluation in Counseling and Development, 38, 78-91.

Burnham, J. J. (2009). Contemporary fears of children and adolescents: Doping and resiliency in the 21 st century. Journal of Counseling and Development, 87(1), 28-35.

Cangas, A. J., Gázquez, J. J., Pérez-Fuentes, M. C., Padilla, D. y Miras, F. (2007). La evaluación de la violencia escolar y su afectación personal en una muestra de estudiantes europeos. Psicothema, 19, 114-119.

Castro, B. A. y Gaviria, M. B. (2005). Clima escolar y comportamientos psicosociales en niños. Revista de la Facultad Nacional de Salud Pública, 23(2), 59-69.

Comer, J. P., Ben-Avie, M., Haynes, N. M. y Joyner, E. T. (1999). Child by child. The Comer process for change in education. Nueva York: Teachers Collage Press.

Dunn, J. A. (1970, septiembre). The School Anxiety Questionnaire: Theory, instrument and summary of results. Presentado en American Psychological Association Convention. Miami, EEUU.

Elgar, F., Arlett, C. y Groves, R. (2003). Stress, coping and behavioural problems among rural and urban adolescents. Journal of Adolescence, 26, 574-585. 
Emmons, C. L., Comer, J. P. y Haynes, N. M. (1996). Translating theory into practice: Comer's theory of school reform. En J. P. Comer, N. M. Haynes, E. Joyner y M. Ben-Avie (Eds.), Rallying the whole village (pp. 27-41). Nueva York: Teachers College Press.

Escobar, M., Blanca, M. J., Fernández-Baena, F. J. y Trianes, M.V. (2011). Adaptación española de la escala de manifestaciones de estrés del Student Stress Inventory (SSI-SM). Psicothema, 1-12.

Escobar, M., Trianes, M. V., Fernández, F. J. y Miranda, J. (2010). Relaciones de la aceptación sociométrica con inadaptación socioemocional, estrés cotidiano y afrontamiento en escolares considerando diferencias de género y edad. Revista Latinoamericana de Psicología, 42, 469-479.

Espinoza, E. (2006). Impacto del maltrato en el rendimiento académico. Electronic Journal of Research in Educational Psychology, 4(2), 221-238.

Ezpeleta, L. (2005). Factores de riesgo en psicopatología del desarrollo. Barcelona: MassonSalvat.

Felner, R. D., Brand, S., DuBois, D. L. Adan, A. M., Mulhall, P. F. y Evans, E. G. (1995). Socioeconomic disadvantage, proximal environmental experiences and socioemotional and academic adjustment in early adolescence: Investigation of a mediated effects model. Child Development, 66, 774-792.

Fernández-Baena, F. J., Trianes, M. V., de la Morena, M. L., Escobar, M., Infante, L. y Blanca, M. J. (2011). Propiedades psicométricas de un cuestionario para la evaluación de la violencia cotidiana entre iguales en el contexto escolar. Anales de Psicología, 27(1), 102-108.

García-Fernández, J. M., Espada, J. P., Orgilés, M. y Méndez, F. X. (2010). Psychometric properties of the School Fears Survey Scale for preadolescents (SFSS-II). Psicothema, 22(3), 502-508.

García-Fernández, J. M., Inglés, C. J., Martínez-Monteagudo, M. C., Marzo, J. C. y Estévez, E. (2011). Inventario de Ansiedad Escolar: validación en una muestra de estudiantes de educación secundaria. Psicothema, 23(2), 301-307.

García-Fernández, J. M., Inglés, C. J., Martínez-Monteagudo, M. C. y Redondo, J. (2008). Evaluación y tratamiento de la ansiedad escolar en la infancia y la adolescencia. Behavioral Psychology/Psicología Conductual, 16, 413-437.

Gázquez, J. J., Pérez, M. C. y Carrión, J. (2011). Clima escolar y resolución de conflictos según el alumnado: un estudio europeo. Revista de Psicodidáctica, 16(1), 39-58. 
Granell de Aldaz, E., Vivas, E., Gelfand, D. y Feldman, L. (1984). Estimating the prevalence of school refusal and school-related fears. A Venezuelan sample. Journal of Nervous and Mental Disease, 172, 722-729.

Guil, R. y Mestre, J. M. (2004). Violencia escolar: su relación con las actitudes sociales del alumnado y el clima social del aula. Revista Electrónica Iberoamericana de Psicología Social (R.E.I.P.S.), 2(1).

Hair, J. F., Anderson, R. E., Tatham, R. C. y Black, W. C. (1998). Multivariate data analysis (5th ed.). Upper Saddle River, NJ: Prentice-Hall.

Hawker, D. S. y Boulton, M. J. (2000). Twenty years' research on peer victimization and psychological maladjustment: A metaanalytic review of cross-sectional studies. Journal of Child Psychology and Psychiatry, 41, 441-455.

Haynes, N. M., Emmons, C. L. y Ben-Avie, M. (1997). School climate as a factor in student adjustment and achievement. Journal of Educational and Psychological Consultation, 8, 321-329.

Infante, L., Hierrezuelo, L., García, B., Sánchez, A., de la Morena, M. L., Muñoz, A. y Trianes, M. V. (2003). Evaluación de actitudes violentas y clima escolar en situaciones de agresividad en alumnado de secundaria. Psicologia, Saúde y Doencas, 4(2), 277-286.

Kuperminc, G. P., Blatt, S. J. y Leadbeater, B. J. (1997). Relatedness, selfdefinition and early adolescent adjustment. Cognitive Therapy and Research, 21, 59-78.

Kuperminc, G. P., Leadbeater, B. J. y Blatt, S. J (2001). School social climate and individual differences in vulnerability to psychopathology among middle school students. Journal of School Psychology, 39(2), 141-159.

Ladd, G.W. (1990). Having friends, keeping friends, making friends and being like by peers in the classroom: Predictors of children's early school adjustment?. Child Development, 61, 312-331.

Martínez-Rodríguez, J. B. (2004). El miedo escolar de los/las alumnos/as y sus diferentes respuestas. Investigación en la Escuela, 54, 27-38.

Méndez, F. X. (1988). Inventario de Miedos Escolares. Trabajo no publicado. Universidad de Murcia.

Méndez, F. X., García-Fernández, J. M. y Olivares, J. (1996). Miedos escolares: Un estudio empírico en preescolar, EGB y BUP. Ansiedad y Estrés, 2(2-3), 113-118.

Monjas, M. I. (2001). La timidez en la infancia y en la adolescencia. Madrid: Pirámide.

Moreno, D., Estévez, E., Murgui, S. y Musitu, G. (2009). Relación entre el clima familiar y el clima escolar: el rol de la empatía, la actitud hacia la autoridad y la conducta violenta 
en la adolescencia. International Journal of Psychology and Psychological Therapy, 9(1), 123-136.

Morris, L. W., Finkelstein, C. S. y Fisher, W. R. (1976). Components of school anxiety: Developmental trends and sex differences. Journal of Genetic Psychology, 128(1), 49-57.

Ollendick, T. H. (1983). Reliability and validity of the Revised Fear Survey Schedule for Children (FSSC-R). Behavior Research and Therapy, 21, 685-692.

Ortega, R., del Rey, R. y Mora-Merchán, J. (2001). Violencia entre escolares. Concepto y etiquetas verbales que definen el fenómeno del maltrato entre iguales. Revista Interuniversitaria de Formación del Profesorado, 41, 95-113.

Parker, J. G. y Asher, S. R. (1987). Peer relations and later personal adjustment: Are low accepted children at risk?. Psychological Bulletin, 102, 357-389.

Roeser, R. W. y Eccles, J. S. (1998). Adolescents' perceptions of middle school: Relation to longitudinal changes in academic and psychological adjustment. Journal of Research on Adolescence, 8, 123-158.

Romasz, T. E., Cantor, J. H. y Elias, M. J. (2004). Implementation and evaluation of urban school-wide social-emocional learning programs. Evaluation and Program Planning, 27, 89-103.

Rosenblatt, J. y Furlong, M. J. (1997). Assessing the reliability and validity of student selfreports of campus violence. Journal of Youth and Adolescence, 26, 187-201.

Sánchez, A. M., Rivas, M. T. y Trianes, M. V. (2006). Eficacia de un programa de intervención para la mejora del clima escolar: algunos resultados. Electronic Journal of Research in Educational Psychology, 4(2), 353-370.

Sandín, B. (1997). Ansiedad, miedos y fobias en niños y adolescentes. Madrid: Dykinson.

Scherer, M. W. y Nakamura, C. Y. (1968). A Fear Survey Schedule for Children (FSS-FC): A factor analytic comparison with Manifest Anxiety (CMAS). Behaviour Research and Therapy, 6, 173-182.

Spielberger, C. D., Gorsuch, R. L. y Lushene, R. E. (1970). Manual for the State/Trait Anxiety Inventory. Palo Alto, CA: Consulting Psychologists Press (versión española, TEA

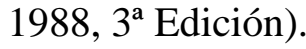

Steinhausen, H. C., Müller, N. y Winkler, C. (2008). Frequency, stability and differentiation of self-reported school fear and truancy in a community sample. Child and Adolescent Psychiatry and Mental Health, 2(1), 1-11. 
Suldo, S. M, Shaunessy, E., Thalji, A., Michalowski, J. y Shaffer, E. (2009). Sources of stress for students in high school college preparatory and general education programs: group differences and associations with adjustment. Adolescence, 44(176), 925-948.

Swartz, K., Reyns, B. W., Henson, B. y Wilcox, P. (2011). Fear of in-school victimization: Contextual, gendered, and developmental considerations. Youth Violence and Juvenile Justice, 9(1), 59-78.

Thomas, S. P. (2010). A novel approach to decreasing bullying. Issues in Mental Health Nursing, 31(9), 551.

Trianes, M. V. (2000). Violencia en contextos escolares. Málaga: Aljibe.

Trianes, M. V. (2004, Octubre). Contextos de la violencia juvenil en España. Ponencia presentada en la VIII Reunión Internacional sobre Biología y Sociología de la Violencia: Violencia y Juventud. Centro Reina Sofía para el Estudio de la Violencia, Valencia, España.

Trianes, M. V., Blanca, M. J., de la Morena, L., Infante, L. y Raya, S. (2006). Un cuestionario para evaluar el clima social del centro escolar. Psicothema, 18(2), 272-277.

Trianes, M. V., Blanca, M. J., Fernández-Baena, F. J., Escobar, M., Maldonado, E. F. y Muñoz, Á. M. (2009). Evaluación del estrés infantil: Inventario Infantil de Estresores Cotidianos (IIEC). Psicothema, 21(4), 598-603.

Valiente, R. M., Sandín, B., Chorot, P. y Tabar, A. (2003). Diferencias según la edad en la prevalencia e intensidad de los miedos durante la infancia y la adolescencia: Datos basados en el FSSC-R. Psicothema, 15(3), 414-419.

Westling, M. (2002). A two level analysis of classroom climate in relation to social context, group composition and organization of special support. Learning Environments Research, 5, 253-274. 